\title{
Band-gaps in long Josephson junctions with periodic phase-shifts
}

\author{
Saeed Ahmad ${ }^{\mathrm{a}}$, Hadi Susanto ${ }^{\mathrm{b}, *}$, Jonathan A. D. Wattis ${ }^{\mathrm{c}}$ \\ ${ }^{a}$ Department of Mathematics, University of Malakand Chakdara, Dir(L), Pakhtunkhwa, Pakistan. \\ ${ }^{b}$ Department of Mathematical Sciences, University of Essex, Colchester CO4 3SQ, UK. \\ ${ }^{c}$ School of Mathematical Sciences, University of Nottingham,University Park, Nottingham NG7 2RD, UK.
}

\begin{abstract}
We investigate analytically and numerically a long Josephson junction on an infinite domain, having arbitrary periodic phase shift of $\kappa$, that is, the so-called 0- $\kappa$ long Josephson junction. The system is described by a one-dimensional sine-Gordon equation and has relatively recently been proposed as artificial atom lattices. We discuss the existence of periodic solutions of the system and investigate their stability both in the absence and presence of an applied bias current. We find critical values of the phase-discontinuity and the applied bias current beyond which static periodic solutions cease to exist. Due to the periodic discontinuity in the phase, the system admits regions of allowed and forbidden bands. We perturbatively investigate the Arnold tongues that separate the region of allowed and forbidden bands, and discuss the effect of an applied bias current on the band-gap structure. We present numerical simulations to support our analytical results.
\end{abstract}

Keywords: Long Josephson junctions, sine-Gordon equation, band-gaps, Arnold tongues

\section{Introduction}

In condensed matter, the tunnelling of Cooper pairs across a barrier between the two superconductors of a long Josephson junction [1] is described by the sine-Gordon equation. In the context of long Josephson junctions, the fundamental topological kink solution of the sine-Gordon model represents a Josephson vortex, or fluxon, that carries a single magnetic flux quantum $\Phi_{0} \approx 2.07 \times 10^{-15}$ Wb. Due to their interesting nonlinear nature, (see, for instance, $[2,3])$ and potential applications $[4,5]$, fluxons have been widely investigated in the last few decades [6].

The vast literature on mathematical models of long Josephson junctions is too far-reaching to cover here;

\footnotetext{
${ }^{*}$ Corresponding author

Email addresses: saeedahmad@uom.edu.pk (Saeed Ahmad), hsusanto@essex.ac.uk (Hadi Susanto)
}

however, Ustinov [7] provides a good review of the wider subject area of solitonic models of Josephson junction. This is firmly based in experimental relevant modelling, and whilst extensive, it is now somewhat dated; it does not cover recent developments such as fractional vortices in systems with phase shifts. In the seminal work [8], McLaughlin and Scott make use of the integrability of the sine-Gordon system to derive formulae for the motion of fluxons in perturbed sine-Gordon systems. Whilst they consider the trajectories which separate pinning from motion, they do not consider fractional fluxons. As well as providing an extensive and relatively uptodate bibliography, Valenti et al. [9] observe that the presence of Gaussian noise in forced and damped sineGordon models of LJJs stabilises the superconducting state; that is, switching times to the resistive state are increased due to stochastic resonance. The effect of thermal fluctuations on the lifetimes of the superconducting metastable states in a long Josephson junc- 
tion with no phaseshifts is considered by Augello et al. [10]. The lifetimes have strong dependence on the frequency of the applied noise and is also influenced by any correlation. Fedorov and Pankratov [11] consider a two-dimensional sine-Gordon system with damping and forcing, which includes stochastic fluctuations but has no phase shift. They find that the mean escape time is significantly longer than the corresponding one-dimensional system. Hence they are able to describe how the escape rate depends on the topological charge of the vortex. The case of overdamped sine-Gordon system with a forcing term is considered by Büttiker and Landauer [12] - this constitutes a considerable simplification to the dynamics. They consider a generic pendulum model, with no specific terms for long Josephson junctions. Federov et al. [13] also consider the switching times of long Josephson junctions with noise in the overdamped limit. They find that in short junctions, noise delays the decay, but that once the lengths exceeds several times the penetration depth, there is no significant effect. The case of a LJJ with dichotomous noise is considered by Guarcello et al. [14], who again observe that noise enhances stability.

In the late seventies, Bulaevskii et al. $[15,16]$ predicted, that it was possible to create half integer Josephson vortices that carry half of the magnetic flux $\left(\Phi_{0} / 2\right)$, often called semifluxons [17]. To do so, one has to use a junction having one part with positive critical current (0-part) and the other with negative critical current ( $\pi$-part), i.e., the so-called $0-\pi$ long Josephson junction. This prediction was later confirmed experimentally, see for example, Kirtley et al.. [18] and Sugimoto et al. [19]. The dynamics of such junctions is described by a model with a discontinuous Josephson phase at the $0-\pi$ boundary.

The possibility of creating an arbitrary (i.e., not only $\pi$ ) phase shift in the Josephson phase $(\phi)$ was successfully fabricated experimentally by Goldobin et al. [20]. If $x$ is the coordinate along the Josephson junction having a $\kappa$-phase discontinuity at $x=0$, then the current-phase relation in the region $x>0$ reduces to $I_{s}=I_{c} \sin (\phi+\kappa)$. Such junctions can be created by a pair of closely situated current injectors, where $\kappa$ and the current through the injectors are found to be proportional [21, 22]. It was demon- strated by Goldobin et al. [23] that the phase shift can be tuned to be of any value $\kappa$ and consequently, an arbitrary fractional magnetic flux quantum, referred to as a fractional vortex, may spontaneously appear in order to compensate the phase jump.

The eigenfrequency of a fractional vortex corresponds to the oscillation of the magnetic flux of the vortex around the point of discontinuity and depends upon the parameter $\kappa$. Goldobin et al. [24] reported that the eigenfrequency of a fractional vortex in a long $0-\kappa$ Josephson junction depends upon the magnetic flux of the vortex and it lies within the band-gap. A later numerical study [25] of a one-dimensional chain of fractional vortices in a long Josephson junctions having alternating $\pm \kappa$ discontinuities focused on the small oscillations of crystal of fractional vortices and the corresponding energy bands in terms of the phase discontinuity $\kappa$. Vogel et al [26] analyse the escape rate of fluxons in a biased $0-\kappa$ long Josephson junction due to thermal and quantum fluctuations. Their analytical work relies on finding the stationary solution, and then determining the stability through a detailed eigenmode calculation. Buckenmaier et al. [27] demonstrate good experimental agreement between the theory of $0-\kappa$ LJJs and spectroscopic measurements, confirming the dependence of eigenfrequency on bias current. However, analytic descriptions of the bifurcation points of the energy bands and the effect of the applied bias current on additional openings of the band-gaps are still lacking. In this paper, we consider a long Josephson junction with a periodic phase jump of arbitrary size $\kappa$ and study the energy bands that correspond to the lattice oscillations analytically as well as numerically.

The study of propagation of a nonlinear wave in periodic structures (e.g., crystals) is a fascinating topic of research in solid state physics, optics and applied mathematics $[28,29,30]$. Periodic structures have important features, including the existence of multiple frequency gaps (also called Bloch bands or bandgaps) in the wave transmission spectra. These bandgaps play an important role in all physical properties of solids. By controlling their periodicity, the basic properties of a material can be altered and a material with desirable properties can be created. This 
process is complicated because of the fixed structure of crystals in nature and the requirement of, for example, a strong electric [31], or magnetic field [32]. To allow the control of the electronic properties of a material to a wider extent, it would be interesting to construct and understand artificial periodic structures with a wide range of varying properties during experiments. Long Josephson junctions with periodic phase shifts have been proposed to be artificial periodic structures with electronic properties that can be controlled up to a greater degree during experiment, through the variation of the current or by choosing the inter-vortex distance at the design time [25]. In the present paper we aim to provide a systematic study of the allowed and forbidden bands of the fractional vortices based periodic structures.

This paper is structured as follows. In Section 2 , we present a mathematical model for the problem under consideration and its temporally stationary and spatially uniform solutions. Section 3 describes the results of a detailed study of the periodic solutions about the uniform solutions including the ground state solutions both in the absence and in the presence of an applied bias current. This section also deals with the dependence of the ground state on a inter-vortex distance parameter $a$, and on the arbitrary periodic discontinuity $\kappa$. In Section 5 , we study the stability of the periodic solutions and derive the expressions of the transitional curves which separate regions of allowed and forbidden bands. We study the points where the band-gaps bifurcate, and study the opening of the additional band-gaps in the presence of the applied bias current. We also find analytic expressions for the corresponding eigenfunctions. Finally, Section 6 concludes our work.

\section{Mathematical Model}

The dynamics of the phase difference between the superconductors of a $0-\kappa$ long Josephson junction is described by the one-dimensional sine-Gordon equation $[17,25]$

$$
\phi_{x x}-\phi_{t t}=\sin [\phi+\theta(x)]-\gamma, \quad(-\infty<x<\infty) \text {. }
$$

This equation is obtained after rescaling where the spatial and the temporal variables are respectively normalized to the Josephson penetration depth $\left(\lambda_{J}\right)$, and the inverse plasma frequency $\left(\omega_{p}^{-1}\right)$. The quantity $\gamma$ represents the applied bias current density which is normalized to critical current density $\left(J_{c}\right)$ of the junction. The function $\theta(x)$, defined by

$$
\theta(x)= \begin{cases}0, & x \in(2 n a,(2 n+1) a), \\ \kappa, & x \in((2 n+1) a, 2(n+1) a),\end{cases}
$$

represents the absence or presence of an additional periodic phase-shift in the Josephson phase. Here, $n \in \mathbb{Z}, 0<\kappa<2 \pi$, and $a$ is the distance between two consecutive phase-discontinuities.

Considering the structure of the additional periodic phase shift, $\theta(x)$, it is convenient to simplify the problem under consideration without losing generality. Thus, to analyse the ground states of an infinitely $0-\kappa$ long Josephson junction with periodic phase shifts, we consider

$$
\phi_{x x}-\phi_{t t}=\sin [\phi+\theta(x)]-\gamma, \quad(0<x<2 a),
$$

where

$$
\theta(x)=\left\{\begin{array}{lc}
0, & (0<x<a), \\
\kappa, & (a<x<2 a) .
\end{array}\right.
$$

For natural purposes, we assume the continuity of the Josephson phase and the magnetic field at $x=a$ and at $x=0,2 a$ under periodic boundary conditions

$$
\begin{array}{cc}
\phi\left(a^{-}\right)=\phi\left(a^{+}\right), & \phi_{x}\left(a^{-}\right)=\phi_{x}\left(a^{+}\right), \\
\phi\left(0^{+}\right)=\phi\left(2 a^{-}\right), & \phi_{x}\left(0^{+}\right)=\phi_{x}\left(2 a^{-}\right) .
\end{array}
$$

The stationary solutions of Eq. (3) are determined by solving its time-independent form

$$
\phi_{x x}=\sin [\phi+\theta(x)]-\gamma
$$

which admits uniform solutions that, for small $\gamma$, and in the regions $0<x<a$, and $a<x<2 a$, can be respectively approximated by $\phi_{0} \approx \gamma, \pi-\gamma$, and $\phi_{0} \approx \gamma-\kappa, \pi-\kappa-\gamma$. It is straightforward to verify that for $\kappa \neq 0$, the uniform solutions satisfy the continuity conditions (5) only when $\gamma=0$ and $\kappa \equiv \pi$ $(\bmod 2 \pi)$. When the continuity conditions are not satisfied, we instead obtain spatially periodic solutions. In the following sections, we study the periodic solutions analytically for small $\kappa$ and $\gamma$. The 
analytical results will then be compared with numerical results that are obtained from solving (6) and (4). We discretise the equation and approximate the second derivative using spectral methods [33]. The resulting coupled nonlinear system is then solved using Newton's method.

\section{Periodic solutions about 0 and $-\kappa$}

We first study the existence of periodic solutions about 0 and $-\kappa$. For this purpose, we perform a perturbation analysis and assume

$$
\phi(x)=\left\{\begin{array}{cc}
\widetilde{\phi}, & (0<x<a), \\
-\kappa+\widehat{\phi}, & (a<x<2 a),
\end{array}\right.
$$

where $|\widetilde{\phi}| \ll 1$ and $|\widehat{\phi}| \ll 1$ are functions of the spatial variable $x$ which are subject to the continuity and boundary conditions

$$
\begin{array}{rlll}
\widetilde{\phi}(a) & =\widehat{\phi}(a)-\kappa, & \widetilde{\phi}(0) & =\widehat{\phi}(2 a)-\kappa, \\
\widetilde{\phi}_{x}(a) & =\widehat{\phi}_{x}(a), & \widetilde{\phi}_{x}(0) & =\widehat{\phi}_{x}(2 a) .
\end{array}
$$

Our aim is to determine these small perturbations in the respective regions.

Inserting the ansatz (7) into the static sine-Gordon equation (6), a formal series expansion up to the leading order of $\widetilde{\phi}$ and $\widehat{\phi}$ yields a set of two ordinary differential equations whose general solutions have the form

$$
\begin{aligned}
& \widetilde{\phi}=\widetilde{A} \cosh \left(x-x_{1}\right)+\gamma, \quad(0<x<a), \\
& \widehat{\phi}=\widehat{A} \cosh \left(x-x_{2}\right)+\gamma, \quad(a<x<2 a),
\end{aligned}
$$

where $|\widetilde{A}| \ll 1,|\widehat{A}| \ll 1$ are constants of integration, and $x_{1}, x_{2}$ are arbitrary points in the domain $0<x<$ $2 a$ which are chosen in order to satisfy the continuity and bounday conditions. However, in the following, we shall consider higher-order correction terms. To do so, we consider two problems.

Firstly, a Taylor series expansion of (6) up to the third correction terms in $\widetilde{\phi}$ and $\widehat{\phi}$ gives

$$
\left\{\begin{array}{cc}
\widetilde{\phi}_{x x}-\widetilde{\phi}+\frac{\widetilde{\phi}^{3}}{6}+\gamma=0, \quad(0<x<a), \\
\widehat{\phi}_{x x}-\widehat{\phi}+\frac{\widehat{\phi}^{3}}{6}+\gamma=0, \quad(a<x<2 a) .
\end{array}\right.
$$

Motivated by the Poincare-Lindstedt method [34], to solve system (10), we propose an ansatz of the form

$$
\begin{aligned}
\widetilde{\phi} & =\widetilde{A} \cosh \left[\widetilde{\omega}\left(x-x_{1}\right)\right]+\gamma,(0<x<a), \\
\widehat{\phi} & =\widehat{A} \cosh \left[\widehat{\omega}\left(x-x_{2}\right)\right]+\gamma,(a<x<2 a),(12
\end{aligned}
$$

where $\widetilde{\omega}=1+\epsilon \widetilde{\omega}_{1}+\mathcal{O}\left(\epsilon^{2}\right), \widehat{\omega}=1+\epsilon \widehat{\omega}_{1}+\mathcal{O}\left(\epsilon^{2}\right)$ with $\widetilde{\omega}_{1}$ and $\widehat{\omega}_{1}$ being constants that are determined later. One may choose $x_{1}=a / 2$ and $x_{2}=3 a / 2$, so as to impose even symmetry about $x=a / 2$ and about $x=3 a / 2$.

The idea of the Poincaré-Lindstedt method is to stretch the $x$-axis, and approximate the solution over one period. Since the Poincaré method is for oscillatory problems, the stretch, $\omega_{1}$, is determined by suppressing the secular terms, see [34] for details. Here, we have a solution which is not in terms of oscillatory (trigonometric) functions, but rather in terms of hyperbolic functions. Hence, there are no secular terms in the present case. Therefore, to find $\omega_{1}$, we need another method so that the leading order solution in terms of elementary functions is a good approximation to the given solution. We introduce the scaling

$$
\widetilde{A}=A \sqrt{\epsilon}, \gamma=\widetilde{\gamma} \sqrt{\epsilon}, a=\mathcal{O}(1) .
$$

Secondly, we want the approximate solutions to be accurate approximations of the trajectories in the phase spaces $(\widetilde{\phi}, \widetilde{\psi})$ and $(\widehat{\phi}, \widehat{\psi})$. In order to solve the system (10) in the region $0<x<a$, we use the ideas behind phase-plane analysis to formulate an equation for $\widetilde{\phi}_{x}=\widetilde{\psi}$ in terms of $\widetilde{\phi}$. After some calculations, one arrives at the differential equation for the phase path

$$
\frac{d \widetilde{\psi}}{d \widetilde{\phi}}=\frac{\widetilde{\phi}-\widetilde{\phi}^{3} / 6-\gamma}{\widetilde{\psi}}
$$

This equation is separable and, on integration, leads to

$$
\frac{\widetilde{\psi}^{2}}{2}=\frac{\widetilde{\phi}^{2}}{2}-\frac{\widetilde{\phi^{4}}}{24}-\gamma \widetilde{\phi}+C .
$$

Here, $C$ is a constant of integration, which determines the trajectory under consideration. For the trajectory we are interested in, $C$ is determined by using $\widetilde{\phi}\left(x_{1}\right)=\widetilde{A}+\gamma$ and $\widetilde{\psi}\left(x_{1}\right)=0$. Once the value 
of $C$ is found, a simple manipulation leads from Eq. tions for the two unknowns $\widetilde{A}$ and $\widehat{A}$, namely

(15) to

$$
\begin{aligned}
\widetilde{\psi}^{2}-\widetilde{\phi}^{2}+\widetilde{A}^{2}+\gamma^{2}+2 \widetilde{A} \gamma \cosh \Psi \\
=\frac{\widetilde{A}}{12}\left\{\widetilde{A}^{3}\left[1-\cosh ^{4} \Psi\right]+4 \widetilde{A}^{2} \gamma\left[1-\cosh ^{3} \Psi\right]\right. \\
\left.\quad+6 \widetilde{A} \gamma^{2}\left[1-\cosh ^{2} \Psi\right]+4 \gamma^{3}[1-\cosh \Psi]\right\}
\end{aligned}
$$

where $\Psi=\widetilde{\omega}(x-a / 2)$.

Next, by discarding smaller terms in $\epsilon$ and using a hyperbolic identity, from Eqs. (11) and $\widetilde{\phi}_{x}=\widetilde{\psi}$, we obtain

$\widetilde{\psi}^{2}-\widetilde{\phi}^{2}+\widetilde{A}^{2}+\gamma^{2}+2 \widetilde{A} \gamma \cosh \Psi=-2 \epsilon \widetilde{\omega}_{1} \widetilde{A}^{2} \sinh ^{2} \Psi$.

By comparing Eqs. (16) and (17), one would normally need to require the right hand sides of the two equations to be the same. However, they are different and the difference is dependent on the spatial variable $x$. This matter arises because we require an approximate solution to satisfy the governing equation. An optimal way of making them equal is by averaging the difference, i.e. integrate the resulting equation over the interval $0<x<a$ and divide the integrand by the length of the interval, and requiring this spatial average difference to be zero. This approach leads to an implicit relation, which with the help of the scalings (13) and a formal series expansion to the leading order of $\epsilon$ gives

$$
\begin{aligned}
\widetilde{\omega}_{1}= & -\frac{1}{24 a}\left\{\frac{A^{2}}{2}(3 a+\sinh (a))+8 A \widetilde{\gamma} \sinh (a / 2)\right. \\
& \left.+6 \widetilde{\gamma}^{2} a+8 \widetilde{\gamma}\left(\frac{A^{2}+\widetilde{\gamma}^{2}}{A}\right) \tanh (a / 4)\right\} . \quad(18)
\end{aligned}
$$

Following the same steps, by taking as the general solution of the second equation of the system (10) in the region $a<x<2 a$, one may verify that $\widehat{\omega}_{1}$ is given by the same equation as (18), but the parameter $A$ is now the corresponding amplitude of the phase in the region $a<x<2 a$.

Next, we apply the conditions (5) to the general solution of the system, and obtain a set of two equa-

$$
\left\{\begin{array}{l}
(\widetilde{A}-\widehat{A}) \cosh \left(\left(1+\epsilon \widetilde{\omega}_{1}\right) \frac{a}{2}\right)+\kappa=0, \quad(0<x<a), \\
(\widetilde{A}+\widehat{A}) \sinh \left(\left(1+\epsilon \widehat{\omega}_{1}\right) \frac{a}{2}\right)=0, \quad(a<x<2 a) .
\end{array}\right.
$$

The second equation of the system (19) holds if $\widetilde{A}=$ $-\widehat{A}$. As a result, the first equation of the system (19) reduces to

$$
\widetilde{A} \cosh \left(\left(1+\epsilon \widetilde{\omega}_{1}\right) a / 2\right)=-\kappa / 2 .
$$

Let $\kappa=\sqrt{\epsilon} \widetilde{\kappa}$. Introducing the scaling (13) into (20), a Taylor series expansion about $\epsilon=0$ with smaller terms being neglected leads to

$$
\begin{aligned}
A= & -\frac{\widetilde{\kappa}}{2 \cosh (a / 2)}-\frac{A_{1}}{768 \cosh ^{4}(a / 2)} \\
A_{1}= & \widetilde{\kappa}^{3}(3 a+\sinh (a)) \sinh (a / 2) \\
& -16 \widetilde{\kappa}^{2} \widetilde{\gamma} \sinh (a)\{3 \tanh (a / 4)+\sinh (a / 2)\} \\
& -768 \widetilde{\gamma}^{3} \operatorname{sech}^{2}(a / 4) \cosh ^{3}(a / 2)
\end{aligned}
$$

We are now in position to write the periodic solution of the system $(10)$ in the driven case $(\gamma \neq 0)$ in the form

$$
\phi(x)=\left\{\begin{array}{c}
\widetilde{A} \cosh \left(\left(1+\epsilon \omega_{1}\right)(x-a / 2)\right)+\gamma \\
(0<x<a), \\
-\kappa-\widetilde{A} \cosh \left(\left(1+\epsilon \omega_{1}\right)(x-3 a / 2)\right)+\gamma, \\
(a<x<2 a) .
\end{array}\right.
$$

Here, $\omega_{1}=\widetilde{\omega}_{1}$.

In Fig. 1, we have depicted the profile of the Josephson phase $\phi$ of of the system (3)-(4) as a function of the spatial variable $x$. The solid lines represent the numerically obtained solution for $a=1$ and $\kappa=0.5$, while the dashed lines represent the corresponding analytical approximation (22).

First we study the behaviour of the wave function $\phi(x)$ in the absence of an applied bias current $\gamma$ (see the top panel of Figure 1). One can observe that 

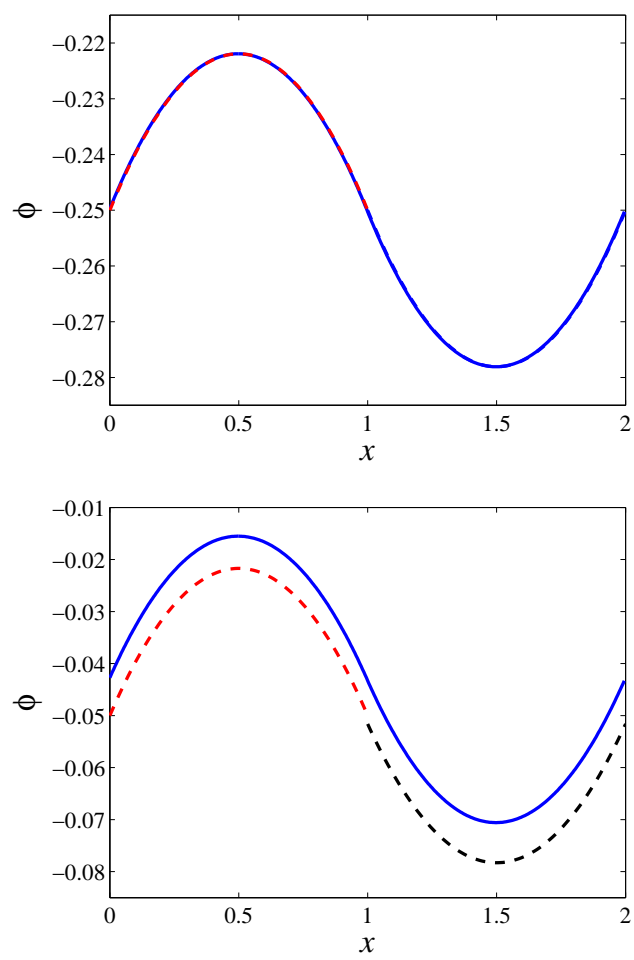

Figure 1: Comparison of the Josephson phase $\phi$ obtained from solving (6) and (4) numerically (solid lines) and the corresponding approximations (dotted lines) of the profile $\phi$ given by the system (22) in terms of the spatial variable $x$ for the undriven (top) and the driven (bottom) cases. In the driven case we have taken $\gamma=0.2$. Here, the inter-vortex distance, $a$, is taken to be unity and $\kappa=0.5$ in both the driven and undriven problems. The value of the discontinuity $\kappa$ is 0.5 .
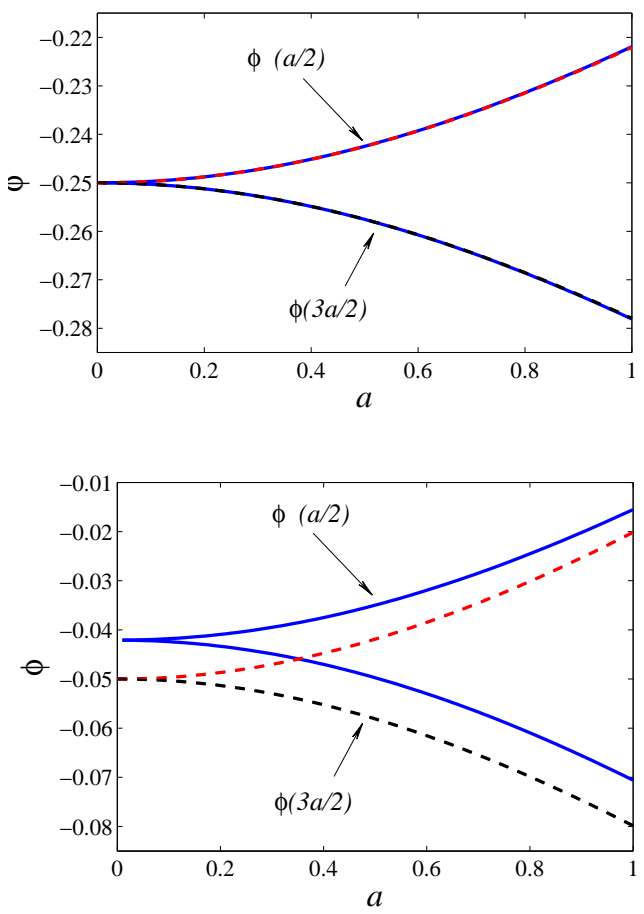

Figure 2: Plot of the approximations (dashed lines) to the profile $\phi(a / 2)$ and $\phi(3 a / 2)$, given by the system $(22)$ at $\kappa=$ 0.5 , as a function of the facet length $a$ for the case of $\gamma=0$ (top) and $\gamma=0.2$ (bottom). Solid lines represent the numerically obtained periodic solutions of (6) with (4).

$\phi(x)$ oscillates and attains its maximum and minimum values respectively at the points $x=a / 2$ and $x=3 a / 2$. We observe good agreement between the approximation and the corresponding numerics. The lower panel of the same figure, shows the periodic solution $\phi(x)$ plotted against $x$ in the driven situation, where we take $\gamma=0.2$. We also note that the approximation of the undriven case is closer to numerics than the driven case. Differences in the driven case are $\sim 0.01$ which is $\mathcal{O}\left(\epsilon^{3}\right)$, given $\gamma=0.2$.

The approximations of the Josephson phase $\phi(x)$ given by the system (22) at $x=a / 2$ (the upper branch) and $x=3 a / 2$ (the lower branch) as functions of the parameter $a$ are shown in Fig. 2. The upper and lower panels represent the undriven and driven cases respectively. Consider the top panel of 


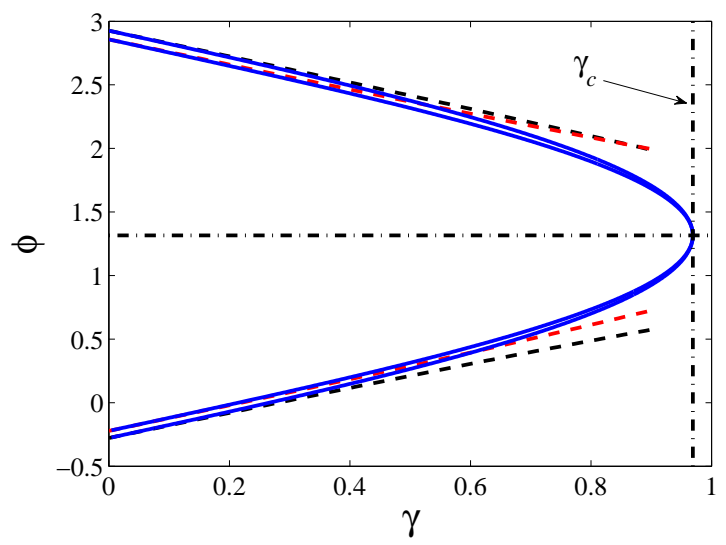

Figure 3: Comparison of the approximations to the profile of the wavefunctions $\phi(a / 2)$ and $\phi(3 a / 2)$, (dotted lines) given by the systems (22) and (24) and the corresponding numerical solution (solid lines) as a function of the applied bias current $\gamma$ for $a=1$ and $\kappa=0.5 . \quad \gamma_{c}$ denotes the critical current at which a turning point in the existence curve occurs.

the figure; when $a=0$, the two branches merge at the point $\phi \approx-0.25$, which shows that the magnetic field $\phi_{x}$ disappears. Observe that the strength of the magnetic field intensity is related proportionally to inter-vortex distance in Josephson junction. We observe a rather good agreement between the approximations and numerics in this case. In the lower panel of the same figure, the profile of the superconducting phase $\phi$ as a function of $a$ is plotted in the presence of the external force, where we have taken $\gamma=0.2$. Again, one can observe that the magnetic flux is zero at $\phi \approx-0.041$, and increases with increasing $a$. A comparison between our approximations and the corresponding numerics is displayed in the same figure showing good qualitative agreement.

The effect of the applied bias current, $\gamma$, on the profile of the Josephson phase $\phi$ given by the system (22) is displayed by the lower dashed lines in Figure 3. The two lower branches correspond to $x=a / 2$ and $x=3 a / 2$. The Josephson phase $\phi$ increases with the increase in the bias current $\gamma$. For $\gamma$ close to zero, the approximation to the periodic solution, given by (22), approximates well its numerical counterpart.

When $\gamma$ is increased further, a particular value of the bias current is reached where the solution ceases to exist due to a turning point. Here, we have a saddle-node bifurcation between the periodic solutions about 0 and $-\kappa$ and those about $\pi$ and $\pi-\kappa$ that we will discuss in the section below. This particular value of the bias current is denoted by $\gamma_{c}$. We have found that the value of $\gamma_{c}$ depends on the parameter $\kappa$ and the facet length $a$. For $a=1$ and $\kappa=0.5$, we have $\gamma_{c} \approx 0.96$.

\section{Periodic solutions about $\pi$ and $\pi-\kappa$}

Next, we consider periodic solutions about $\phi=$ $\pi, \pi-\kappa$. In this case, our perturbation ansatz has the form

$$
\phi=\left\{\begin{array}{rc}
\pi+\tilde{\phi}, & (0<x<a) \\
\pi-\kappa+\widehat{\phi}, & (a<x<2 a)
\end{array}\right.
$$

where $|\widetilde{\phi}| \ll 1$ and $|\widehat{\phi}| \ll 1$ are functions of the spatial variable. Following steps similar to the previous section, we find that the periodic solutions of the system about $\pi$ and $\pi-\kappa$ in the driven case have the form

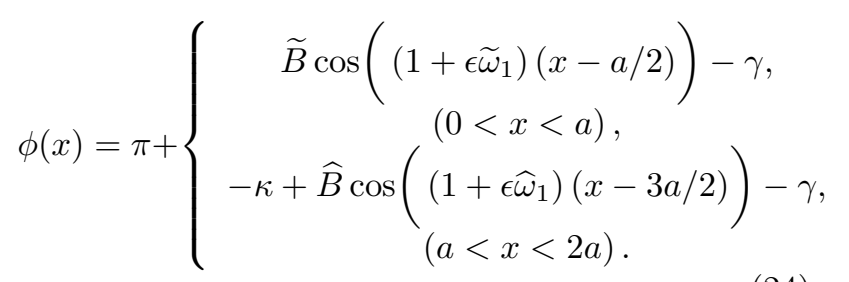

Using the scaling of the previous section along with $\widetilde{B}=\sqrt{\epsilon} B, \widetilde{\omega}_{1}$ can be calculated as

$$
\begin{aligned}
\widetilde{\omega}_{1}= & \frac{-1}{48 a}\left\{B^{2}(3 a+\sin (a))-16 B \widetilde{\gamma} \sin (a / 2)\right. \\
& \left.+12 \widetilde{\gamma}^{2} a-16 \tilde{\gamma}\left(\frac{\widetilde{\gamma}^{2}}{B}+B\right) \tan (a / 4)\right\},(25)
\end{aligned}
$$

while $\widehat{\omega}_{1}$ is the same as $\widetilde{\omega}_{1}$ with the amplitude $B$ is now from the corresponding phase in the second region $a<x<2 a$.

The amplitudes $\widetilde{B}$ and $\widehat{B}$ are related by $\widetilde{B}=-\widehat{B}$, 

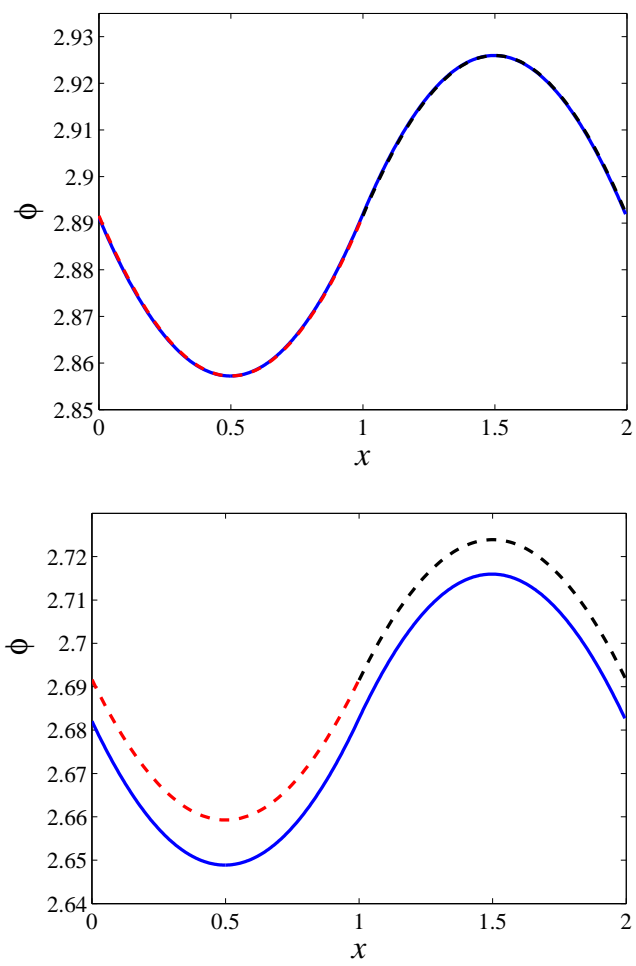

Figure 4: The same as Fig. 1, but for periodic solutions about $\pi$ and $\pi-\kappa$. The approximations are given by system (24). The parameter values as the same as in Fig. 1.

and

$$
\begin{aligned}
B= & -\frac{\widetilde{\kappa}}{2 \cos (a / 2)}+\frac{B_{1} \sin (a / 2)}{768 \cos ^{4}(a / 2)} \\
B_{1}= & \widetilde{\kappa}^{3}(3 a+\sin (a)) \\
& +32 \widetilde{\gamma} \widetilde{\kappa}^{2} \cos (a / 2)\{\tan (a / 4)+\sin (a / 2)\} \\
& +16 \gamma^{2}(8 \widetilde{\gamma} \tan (a / 4) \cos (a / 2)+3 a \widetilde{\kappa}) \\
& \times \cos ^{2}(a / 2) .
\end{aligned}
$$

First, the profiles of the wave function $\phi$ in terms of the spatial variable $x$ is depicted in Fig. 4. The parameter values are the same as those in Fig. 1. The periodic in space $\phi$ attains its minimum and maximum values respectively at $x=a / 2$ and $x=3 a / 2$. A very close agreement between the analytical calculations (24) and the corresponding numerics is ob-
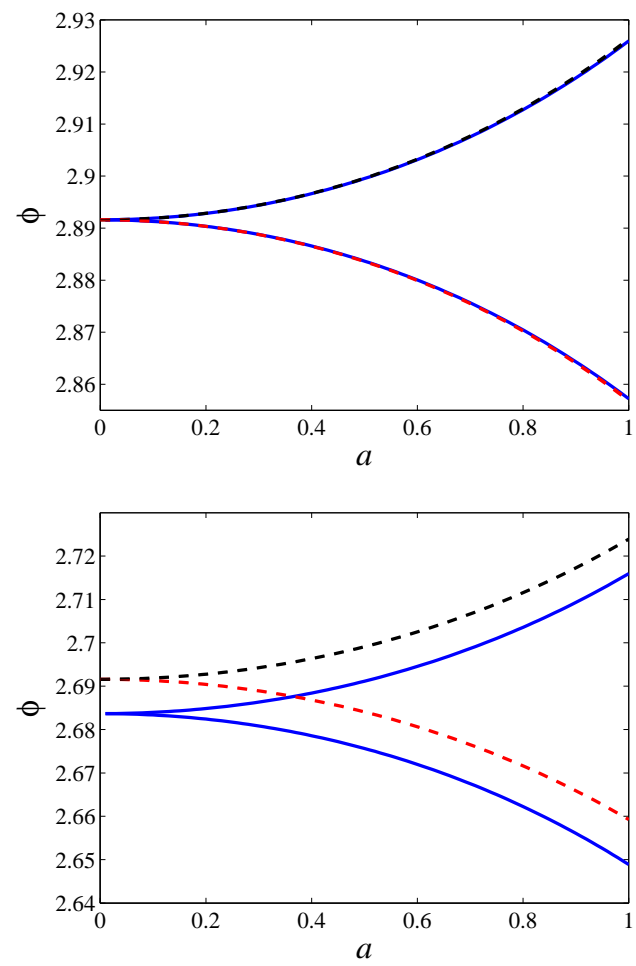

Figure 5: The same as Fig. 2, but for periodic solutions about $\pi$ and $\pi-\kappa$. The approximations (dashed lines) are given by system (24).

served.

The behaviour of $\phi$ as a function of the facet length $a$ in the absence and presence of the applied bias current are shown in upper and lower panels of Fig. 5. The magnetic flux is zero when $a$ is zero and increases with increasing $a$.

Next, we study the periodic solutions of the system about $\pi$ and $\pi-\kappa$ for varying applied bias current. The portion above the line $\phi \approx 1.31$ in Fig. 3 depicts the profile of the Josesphson phase $\phi$ as function of the applied bias current $\gamma$. The lower and upper branches respectively correspond to the value of the Josephson phase at $x=a / 2$ and $x=3 a / 2$. The gap between the two branches decreases with the increase in bias current $\gamma$. It is clear from the figure that the periodic solutions about 0 and $\pi$ are symmetric about the horizontal line $\phi \approx 1.31$. For a small $\gamma$ our an- 

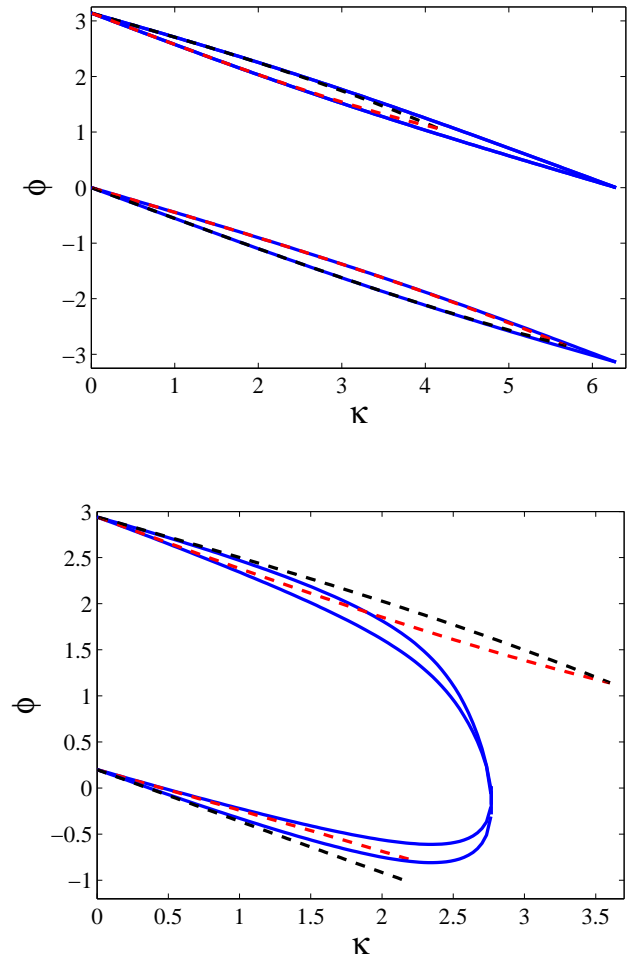

Figure 6: Plots of the approximations to the profile $\phi$, given by the systems (22) and (24) as a function of the parameter $\kappa$. The upper panel is when there is no bias current applied to the system, i.e. $\gamma=0$, and the lower panel shows the case when $\gamma=0.2$.

alytical results (22) and (24) well approximate their corresponding numerical counterparts.

As mentioned above, there is a critical value of bias current called $\gamma_{c}$ above which the periodic solutions cease to exist. Note that one of the two kinds of periodic solutions discussed herein will be the ground state of the time-dependent governing equation (3). The presence of the critical bias current means that the static ground state of the system can disappear and one obtains a time-dependent state instead, as reported previously in [35].

Figure 6 shows the profile of the wave function $\phi$ given by (22) and (24) as a function of the parameter $\kappa$. In the undriven case where $\gamma=0$, there are two solutions emanating from $\phi=0$ and $\phi=\pi$ on the axes where $\kappa=0$, see the upper panel. For the $\pi$-state (0-state), the upper(lower) and lower(upper) branches correspond to $x=a / 2$ and $x=3 a / 2$.

The profile of the Josephson phase $\phi$ as function of $\kappa$ for both periodic solutions about 0 and $\kappa$ in the presence of bias current is shown in the lower panel of Fig. 6, where we have taken $\gamma=0.2$. As $\kappa$ increases further, a stage comes where the two solutions combine via a saddle node bifurcation at a critical value of $\kappa$ denoted by $\kappa_{c}$. For different values of the applied bias current $(\gamma)$ and the facet length parameter $a$, there exists a different $\kappa_{c}$. For example $\kappa_{c}=2.675$, for $a=1$ and $\gamma=0.2$. There is no static solution in the region $\kappa_{c}<\kappa<2 \pi-\kappa_{c}$, in a similar situation as the case when one fixes $\kappa$ and takes $\gamma>\gamma_{c}$ mentioned above (see [35]).

\section{Stability analysis of the periodic solutions}

Next, we study the stability of the periodic solutions about the static solutions and perform perturbation analysis.

To determine the stability of a periodic solution $\phi_{s}$, we assume

$$
\phi(x)=\phi_{s}+\varepsilon e^{\lambda t} V(x), \quad(0<x<2 a),
$$

where $|\varepsilon| \ll 1$ is perturbation parameter and $\lambda \in$ C. Introducing the assumption (27) into the sine Gordon model (3), expanding the resulting equation by Taylor's series and neglecting smaller terms leads to a general eigenvalue problem in the form of the linear Schrödinger equation

$$
V_{x x}-\lambda^{2} V=\cos \left(\phi_{s}+\theta\right) V
$$

From the stability ansatz (27), we say that a solution is (linearly) stable whenever $E=\lambda^{2}<0$ and is unstable otherwise.

To solve the eigenvalue problem (28) numerically, we use spectral methods (see [33], Chapter 9), where $\phi_{s}$ has been computed previously as outlined in Section 2 above. 
5.1. Stability analysis of the periodic solutions about 0 and $-\kappa$

To study the stability of the periodic solutions about the static solutions 0 and $-\kappa, \phi_{s}$ are approximately given by the system (22). In order to simplify our problem, we write the potential in the trigonometric series

$$
\begin{aligned}
\cos \left(\phi_{s}+\theta\right)= & \frac{a_{0}}{2}+R_{1} \sin \left(\frac{\pi x}{a}\right)+R_{2} \cos \left(\frac{2 \pi x}{a}\right) \\
& +R_{3} \sin \left(\frac{3 \pi x}{a}\right)+R_{4} \cos \left(\frac{4 \pi x}{a}\right) .
\end{aligned}
$$

Here the series is truncated at the fourth term and the Euler constants are

$$
\begin{aligned}
& a_{0}=2-\gamma^{2}-\frac{\widetilde{A}^{2}}{2}\left(1+\frac{\sinh (\omega a)}{\omega a}\right) \\
& R_{1}=-\frac{4 \pi \widetilde{A} \gamma \cosh (\omega / 2)}{\pi^{2}+\omega^{2} a^{2}} \\
& R_{2}=-\frac{2 \widetilde{A}^{2} \omega a \sinh (\omega a)}{4 \pi^{2}+4 \omega^{2} a^{2}} \\
& R_{3}=-\frac{12 \pi \widetilde{A} \gamma \cosh (\omega / 2)}{9 \pi^{2}+\omega^{2} a^{2}} \\
& R_{4}=-\frac{2 \widetilde{A}^{2} \omega a \sinh (\omega a)}{16 \pi^{2}+4 w^{2} a^{2}}
\end{aligned}
$$

where $\omega=1+\epsilon \omega_{1}$ and $\omega_{1}$ is given by Eq. (25).

Substituting Eq. (29) into (28), the eigenvalue problem reduces to Mathieu's equation

$$
\begin{array}{r}
V_{x x}-\left[E+\frac{a_{0}}{2}+R_{1} \sin \left(\frac{\pi x}{a}\right)+R_{2} \cos \left(\frac{2 \pi x}{a}\right)+\right. \\
\left.R_{3} \sin \left(\frac{3 \pi x}{a}\right)+R_{4} \cos \left(\frac{4 \pi x}{a}\right)\right] V=0 .
\end{array}
$$

By taking $E+1=-\delta$ and introducing the scaling $R_{i}=-\epsilon r_{i}$, where $i=1,2, \ldots$, Eq. (31) is converted into the canonical form of the Mathieu Equation

$$
\begin{array}{r}
V_{x x}+\left[\delta+\epsilon\left\{E_{0}+r_{1} \sin (\pi x a)+r_{2} \cos \left(\frac{2 \pi x}{a}\right)+\right.\right. \\
\left.\left.r_{3} \sin \left(\frac{3 \pi x}{a}\right)+r_{4} \cos \left(\frac{4 \pi x}{a}\right)\right\}\right] V=0,
\end{array}
$$

where $E_{0}=\frac{1}{2}\left[\widetilde{\gamma}^{2}+\frac{A^{2}}{2}\left(1+\frac{\sinh (\omega a)}{\omega a}\right)\right]$. The general theory (Floquet Theory) of the differential equations with periodic coefficients divides the $(\delta, \kappa)$ plane into the regions of boundedness (stability) and unboundedness (instability) of $V(x)$ as $x \rightarrow \infty$ (see e.g., [36]). The curves separating the regions of stability and instability are known as transition curves or Arnold tongues. The Floquet theory also confirms the existence of linearly increasing and periodic solutions (having periods $2 a$ and $4 a$ ) along the transition curves. In the following we focus on obtaining approximate expressions of the Arnold tongues and the corresponding eigenfunctions $V$. We note that when $\epsilon=0$, the necessary condition for the potential $V$ to be $4 a$-periodic is $\delta=\left(\frac{n \pi}{2 a}\right)^{2}$, where $n \in \mathbf{Z}$. In other words the Arnold tongues intersect $\epsilon=0$ at the critical points

$$
\delta_{c}=\left(\frac{n \pi}{2 a}\right)^{2}, \quad n=0,1,2, \ldots
$$

We follow a perturbation technique, the so called method of strained parameters [37], to investigate the boundary of stability of the periodic solutions. The approximations we find hold only on the transitional curves and hence do not give solution that holds in a small neighborhood of the Arnold tongues.

As discussed earlier, there exist periodic solutions of Eq. (32) on the transition curves having period $2 a$ and $4 a$. These periodic solutions and the transitional curves are determined in the form of the perturbation series

$$
\begin{aligned}
V & =V_{0}+\epsilon V_{1}+\epsilon^{2} V_{2}+\mathcal{O}\left(\epsilon^{3}\right), \\
\delta & =\left(\frac{n \pi}{2 a}\right)^{2}+\epsilon \delta_{1}+\epsilon^{2} \delta_{2}+\mathcal{O}\left(\epsilon^{3}\right) .
\end{aligned}
$$

Putting Eqs. (34) and (35) into Eq. (32) and comparing the coefficients of $\epsilon$ on both sides we obtain

$$
\begin{array}{ll}
\mathcal{O}\left(\epsilon^{0}\right): & V_{0}^{\prime \prime}+\left(\frac{n \pi}{2 a}\right)^{2} V_{0}=0, \\
\mathcal{O}\left(\epsilon^{1}\right): & V_{1}^{\prime \prime}+\left(\frac{n \pi}{2 a}\right)^{2} V_{1}=-S V_{0}, \\
\mathcal{O}\left(\epsilon^{2}\right): & V_{2}^{\prime \prime}+\left(\frac{n \pi}{2 a}\right)^{2} V_{2}=-\delta_{2} V_{0}-S V_{1},
\end{array}
$$


where the primes denote derivatives with respect to $x$ and

$$
\begin{aligned}
S= & \delta_{1}+E_{0}+r_{1} \sin \left(\frac{\pi x}{a}\right)+r_{2} \cos \left(\frac{2 \pi x}{a}\right) \\
& r_{3} \sin \left(\frac{3 \pi x}{a}\right)+r_{4} \cos \left(\frac{4 \pi x}{a}\right) .
\end{aligned}
$$

Below, we find the required expressions for the first few values of $n$.

\subsection{Transitional curves corresponding to $n=0$}

In this case, the periodic particular solution of Eq. (36) is given by $V_{0}=A_{0}$, where $A_{0}$ is a constant of integration. Hence, Eq. (37) becomes

$$
\begin{aligned}
V_{1}^{\prime \prime}= & -A_{0}\left[\delta_{1}+E_{0}+r_{1} \sin \left(\frac{\pi x}{a}\right)+r_{2} \cos \left(\frac{2 \pi x}{a}\right)\right. \\
& \left.+r_{3} \sin \left(\frac{3 \pi x}{a}\right)+r_{4} \cos \left(\frac{4 \pi x}{a}\right)\right] .
\end{aligned}
$$

As $V_{1}$ is expected to be periodic, we have to suppress the secular terms in the last equation. To do so, we must have to take $A_{0}\left(\delta_{1}+E_{0}\right)=0$, giving us two possibilities, viz $A_{0}=0$, or $\delta_{1}+E_{0}=0$. We are not interested in the first possibility as it will make $V_{0}$ trivial. To avoid $V_{0}$ being trivial, we consider only the possibility $\delta_{1}+E_{0}=0$.

Integrating Eq. (41) twice with respect to $x$, and taking $\delta_{1}=-E_{0}$, the periodic particular solution is given by

$$
\begin{aligned}
V_{1}=A_{0} & \left(\frac{a}{\pi}\right)^{2}\left[r_{1} \sin \left(\frac{\pi x}{a}\right)+\frac{r_{2}}{4} \cos \left(\frac{2 \pi x}{a}\right)\right. \\
& \left.+\frac{r_{3}}{9} \sin \left(\frac{3 \pi x}{a}\right)+\frac{r_{4}}{16} \cos \left(\frac{4 \pi x}{a}\right)\right] .
\end{aligned}
$$

Substituting $V_{0}=A_{0}$ and (42) into Eq. (38) and simplifying we may write

$$
\begin{array}{r}
V_{2}^{\prime \prime}=-\delta_{2} A_{0}+A_{0}\left(\frac{a}{\pi}\right)^{2}\left[-S_{1}+S_{2} \sin \left(\frac{\pi x}{a}\right)\right. \\
\left.+S_{3} \cos \left(\frac{2 \pi x}{a}\right)+S_{4} \sin \left(\frac{3 \pi x}{a}\right)+S_{5} \cos \left(\frac{4 \pi x}{a}\right)\right] .
\end{array}
$$

The $S_{i}$ terms are given in Appendix A. The periodicity condition for $V_{2}$ requires

$$
-\delta_{2} A_{0}-A_{0}\left(\frac{a}{\pi}\right)^{2} S_{1}=0,
$$

giving $\delta_{2}=-(a / \pi)^{2} S_{1}$, as $A_{0} \neq 0$. Consequently Eq. (35) becomes

$$
\delta=-\epsilon E_{0}-\epsilon^{2}\left(\frac{a}{\pi}\right)^{2} S_{1} .
$$

After the secular terms have been removed, the periodic solution of Eq. (43) becomes

$$
\begin{array}{r}
V_{2}=A_{0}\left(\frac{a}{\pi}\right)^{4}\left[S_{2} \sin \left(\frac{\pi x}{a}\right)+\frac{S_{3}}{4} \cos \left(\frac{2 \pi x}{a}\right)\right. \\
\left.+\frac{S_{4}}{9} \sin \left(\frac{3 \pi x}{a}\right)+\frac{S_{5}}{16} \cos \left(\frac{4 \pi x}{a}\right)\right] .
\end{array}
$$

By inserting $V_{0}=A_{0}$ and (42) into Eq. (34), the corresponding equation for $V$ can be found.

\subsection{Arnold tongues bifurcating from $\delta=(\pi / 2 a)^{2}$}

In this case the periodic solution of Eq. (36) is given by

$$
V_{0}=A_{0} \cos \left(\frac{\pi x}{2 a}\right)+B_{0} \sin \left(\frac{\pi x}{2 a}\right) .
$$

Substituting this into Eq. (37), one may write

$$
\begin{aligned}
V_{1}^{\prime \prime}+ & \left(\frac{\pi}{2 a}\right)^{2} V_{1} \\
= & -\left[A_{0}\left(\delta_{1}+E_{0}\right)+\frac{r_{1}}{2} B_{0}\right] \cos \left(\frac{\pi x}{2 a}\right) \\
& -\left[B_{0}\left(\delta_{1}+E_{0}\right)+\frac{r_{1}}{2} A_{0}\right] \sin \left(\frac{\pi x}{2 a}\right) \\
& +\frac{1}{2}\left[\left(r_{1} B_{0}-r_{2} A_{0}\right) \cos \left(\frac{3 \pi x}{2 a}\right)\right. \\
& -\left(r_{1} A_{0}-r_{2} B_{0}\right) \sin \left(\frac{3 \pi x}{2 a}\right) \\
& -\left(r_{2} A_{0}+r_{3} B_{0}\right) \cos \left(\frac{5 \pi x}{2 a}\right) \\
& -\left(r_{2} B_{0}+r_{3} A_{0}\right) \sin \left(\frac{5 \pi x}{2 a}\right) \\
& +\left(r_{3} B_{0}-r_{4} A_{0}\right) \cos \left(\frac{7 \pi x}{2 a}\right) \\
& \left.-\left(r_{3} A_{0}-r_{4} B_{0}\right) \sin \left(\frac{7 \pi x}{2 a}\right)\right] .
\end{aligned}
$$

To ensure that $V_{1}$ is periodic, the secular terms must vanish, hence

$$
A_{0}\left(\delta_{1}+E_{0}\right)+\frac{r_{1}}{2} B_{0}=0,
$$




$$
B_{0}\left(\delta_{1}+E_{0}\right)+\frac{r_{1}}{2} A_{0}=0 .
$$

We solve Eq. (49a) for $A_{0}$ which upon substitution into Eq. (49b) gives $\left[\left(\delta_{1}+E_{0}\right)^{2}-\left(r_{1} / 2\right)^{2}\right] B_{0}=0$. For a nontrivial $V_{0}$, one requires $B_{0} \neq 0$, so we are left with $\delta_{1}=-E_{0} \pm r_{1} / 2$. Corresponding to each value of $\delta_{1}$, there is a branch of the transition curve emanating from $\delta=(\pi / 2 a)^{2}$.

5.3.1. The first transition curve bifurcating from $\delta=$ $(\pi / 2 a)^{2}$

Let us first consider the case $\delta_{1}=-E_{0}+r_{1} / 2$. This yields $A_{0}=-B_{0}$ and hence Eq. (47) becomes

$$
V_{0}=-B_{0}\left[\cos \left(\frac{\pi x}{2 a}\right)-\sin \left(\frac{\pi x}{2 a}\right)\right] .
$$

Inserting $V_{0}$ from Eq. (50) into Eq. (48) and integrating the resulting equation twice with respect to $x$, we obtain the particular periodic solution of $V_{1}$ in the form

$$
\begin{aligned}
V_{1}= & -\frac{2^{3 / 2} B_{0} a^{2}}{\pi^{2}}\left[\frac{r_{1}+r_{2}}{9} \sin \left(\frac{3 \pi x}{2 a}+\frac{\pi}{4}\right)\right. \\
& +\frac{r_{2}-r_{3}}{25} \cos \left(\frac{5 \pi x}{2 a}+\frac{\pi}{4}\right) \\
& \left.+\frac{r_{3}+r_{4}}{49} \sin \left(\frac{7 \pi x}{2 a}+\frac{\pi}{4}\right)\right] .
\end{aligned}
$$

with the aid of Eqs. (50) and (51), from Eq. (38), we obtain

$$
\begin{aligned}
V_{2}^{\prime \prime}+ & \frac{\pi^{2} V_{2}}{4 a^{2}}= \\
& \frac{\sqrt{2} a^{2} B_{0}}{\pi^{2}}\left\{\left(S_{6}-\frac{\pi^{2} \delta_{2}}{a^{2}}\right) \cos \left(\frac{\pi x}{2 a}+\frac{\pi}{4}\right)\right. \\
& +S_{7} \sin \left(\frac{3 \pi x}{2 a}+\frac{\pi}{4}\right)+S_{8} \cos \left(\frac{5 \pi x}{2 a}+\frac{\pi}{4}\right) \\
& \left.+S_{9} \sin \left(\frac{7 \pi x}{2 a}+\frac{\pi}{4}\right)\right\}
\end{aligned}
$$

where the $S_{i}$ terms are given in Appendix A.

To ensure that $V_{2}$ is periodic, we take $\delta_{2}=$ $a^{2} S_{6} / \pi^{2}$. By (35), we are now in a position to write the expression for the first transition curve emanating from the point $(\pi / 2 a)^{2}$ as

$$
\delta=\left(\frac{\pi}{2 a}\right)^{2}+\epsilon\left(-E_{0}+\frac{r_{1}}{2}\right)+\epsilon^{2}\left(\frac{a}{\pi}\right)^{2} S_{6} .
$$

Putting the value of $\delta_{2}$ into Eq. (52), the particular solution is obtained by integrating the resulting equation twice with respect to $x$ as

$$
\begin{aligned}
V_{2}= & -\frac{2^{3 / 2} a^{4} B_{0}}{\pi^{4}}\left\{\frac{S_{7}}{9} \sin \left(\frac{3 \pi x}{2 a}+\frac{\pi}{4}\right)\right. \\
& \left.+\frac{S_{8}}{25} \cos \left(\frac{5 \pi x}{2 a}+\frac{\pi}{4}\right)+\frac{S_{9}}{49} \sin \left(\frac{7 \pi x}{2 a}+\frac{\pi}{4}\right)\right\} .
\end{aligned}
$$

By inserting Eqs. (47), (51) and (54) into Eq. (34), the eigenfunction corresponding to the first transition curve becomes

$$
V=V_{0}+\epsilon V_{1}+\epsilon^{2} V_{2}
$$

\subsubsection{The second transitional curve}

Following steps similar to the above, when $\delta_{1}=$ $E_{0}-r_{1} / 2$, we find that $A_{0}=B_{0}$, and

$$
\begin{aligned}
V_{0}= & B_{0} \sin \left(\frac{\pi x}{2 a}+\frac{\pi}{4}\right) \\
V_{1}= & -\frac{2^{3 / 2} a^{2} B_{0}}{\pi^{2}}\left\{\frac{\left(r_{1}-r_{2}\right)}{9} \cos \left(\frac{3 \pi x}{2 a}+\frac{\pi}{4}\right)\right. \\
& -\frac{r_{2}+r_{3}}{25} \sin \left(\frac{5 \pi x}{2 a}+\frac{\pi}{4}\right) \\
& \left.+\frac{r_{3}-r_{4}}{49} \cos \left(\frac{7 \pi x}{2 a}+\frac{\pi}{4}\right)\right\} \\
\delta= & \left(\frac{\pi}{2 a}\right)^{2}-\epsilon\left(E_{0}+\frac{r_{1}}{2}\right)-\epsilon^{2}\left(\frac{a}{\pi}\right)^{2} S_{10} \\
V_{2}= & -\frac{2^{3 / 2} a^{4} B_{0}}{\pi^{4}}\left\{\frac{S_{11}}{9} \cos \left(\frac{3 \pi x}{2 a}+\frac{\pi}{4}\right)\right. \\
& \left.+\frac{S_{12}}{25} \sin \left(\frac{5 \pi x}{2 a}+\frac{\pi}{4}\right)+\frac{S_{13}}{49} \cos \left(\frac{7 \pi x}{2 a}+\frac{\pi}{4}\right)\right\}
\end{aligned}
$$

with $S_{i}$ terms being given in Appendix Appendix A. 

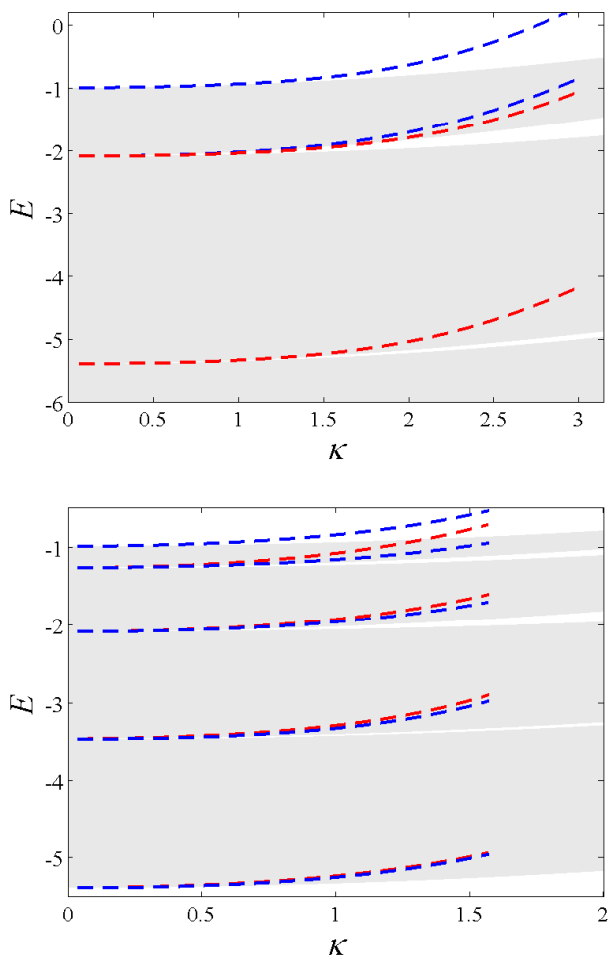

Figure 7: The band-gap structure of the periodic solution about $\phi \equiv 0$ in the undriven $\gamma=0$ (upper panel) and driven $\gamma=0.1$ (lower panel) cases. Dashed lines are given by Eqs. (45), (53), (58), (60), (61), and (62). The boundaries of the grey regions are obtained numerically. Here, we consider a moderate value of inter-vortex distance $a=3$.
One can easily verify that the expressions for the branch of transition curves corresponding to $n=2,3$ and 4 are respectively given by

$$
\begin{aligned}
\delta & =\left(\frac{\pi}{a}\right)^{2}-\epsilon\left(E_{0} \mp \frac{r_{2}}{2}\right)+\epsilon^{2}\left(\frac{a}{\pi}\right)^{2} S_{14}, \\
\delta & =\left(\frac{3 \pi}{2 a}\right)^{2}-\epsilon\left(E_{0} \mp \frac{r_{3}}{2}\right)+\epsilon^{2}\left(\frac{a}{\pi}\right)^{2} S_{23}, \\
\delta & =\left(\frac{2 \pi}{a}\right)^{2}-\epsilon\left(E_{0} \mp \frac{r_{4}}{2}\right)+\epsilon\left(\frac{\pi}{a}\right)^{2} S_{31},
\end{aligned}
$$

with the terms $S_{i}$ being listed in Appendix A.

In Fig. 8 we plot the transition curves given by Eqs. (45), (53), (58), (60), (61), and (62), which divide the $(\delta, \kappa)$ plane into the regions of stability and instability for both vanishing and non-vanishing $\gamma$. Note that in the undrived case $\gamma=0$ we find that $R_{1}=R_{3}=0$. This implies that an application of external current to the junction $(\gamma \neq 0)$ causes the opening of additional band-gaps in the band structure. They emanate from the points $\delta=(n \pi / 2 a)^{2}$. We observe that a good agreement between our approximations and the numerics is only attained near $\kappa=0$.

\subsection{Stability of the periodic solutions about $\pi$ and $\pi-\kappa$}

The stability of the periodic solutions about the static solutions $\pi$ and $\pi-\kappa$ can be studied in a similar fashion. After perturbing the static solutions given by (24) in the form (27), using the analysis and scalings of Section 5.1 with

$$
\begin{aligned}
& a_{0}=-2+\gamma^{2}+\frac{\widetilde{B}^{2}}{2}\left(1+\frac{\sin (\omega a)}{\omega a}\right), \\
& R_{1}=\frac{-2 a \omega \widetilde{B}^{2} \sin (\omega a)}{4 \pi^{2}-4 a^{2} \omega^{2}}, \\
& R_{2}=-\frac{4 \pi \gamma \widetilde{B} \cos \left(\frac{\omega a}{2}\right)}{\pi^{2}-\omega^{2} a^{2}}, \\
& R_{3}=\frac{-2 a \omega \widetilde{B}^{2} \sin (\omega a)}{16 \pi^{2}-4 a^{2} \omega^{2}}, \\
& R_{4}=-\frac{12 \pi \gamma \widetilde{B} \cos \left(\frac{\omega a}{2}\right)}{9 \pi^{2}-\omega^{2} a^{2}}, \\
& E_{0}=\frac{1}{2}\left[\tilde{\gamma}^{2}+\frac{B^{2}}{2}\left(1+\frac{\sin (\omega a)}{\omega a}\right)\right],
\end{aligned}
$$



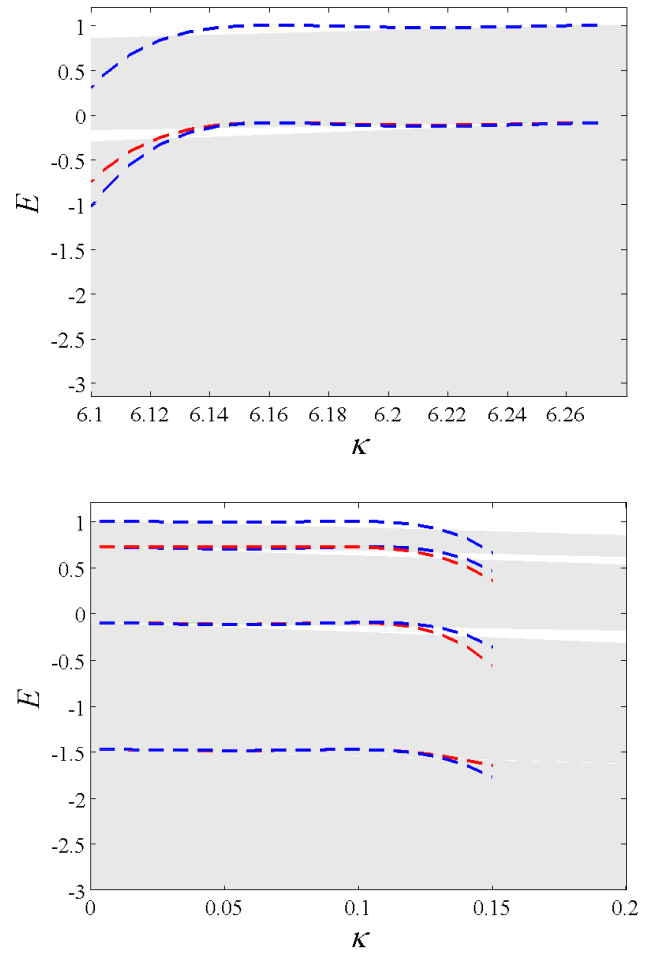

Figure 8: As Fig. 7, but for the corresponding complementary state about $\phi=\pi$. one can derive the same expressions for the Arnold tongues and the corresponding eigenfunctions.

In Fig. 8 we show a comparison between the numerics and our approximation, where we also obtain good agreement between them near $\kappa=0(\bmod 2 \pi)$. Note that even though the horizontal axes of Figs. 7 and 8 may look different, they are actually the same with a transformation. The presentation in figure 8 follows that of Fig. 6, where for $\gamma=0$ the periodic solutions about $\phi=\pi$ are continuations of those about $\phi=0$ at $\kappa=2 \pi$, while for $\gamma \neq 0$ periodic solutions at $\kappa=0$ cannot be continued all the way to $\kappa=2 \pi$ due to a turning-point bifurcation.

\section{Conclusions}

We have considered a long Josephson junction with a periodic phase shift of arbitrary amplitude in the superconducting Josephson phase. Using perturbation techniques, the existence of stationary periodic solutions are discussed both in the absence and presence of an external current. We have demonstrated that the solutions with minimum energy depend upon both the discontinuity $\kappa$ and the facet length $a$. We found that there is a critical value of the applied bias current and the discontinuity, above which each solution will merge into its complementary counterpart.

The magnetic flux of the system is studied in terms of increasing inter-vortex distance in the undriven and driven cases. We have shown that the magnetic flux depends upon the distance between consecutive vortices. The greater the distance between consecutive discontinuities, the larger the magnetic flux is, and vice versa.

When there is no discontinuity in the Josephson phase, the system has a semi-infinite plasma band (or a continuous allowed band) in terms of the spectral parameter $E$. We have shown that, as discontinuities are introduced, one finds forbidden bands in the plasma bands. The expressions for the Arnold tongues which separate an allowed band from a forbidden band have been derived. In addition, it is observed that when one applies an external current to the system, additional band-gaps emanate from the points $E=(n \pi / 2 a)^{2}$, where $n$ is an even integer. These openings expand with the increase in 
the discontinuity $\kappa$. Our results also show that the band-gaps become wider as the distance between discontinuities $(a)$ increases.

It is known that the sine-Gordon model also allows a special type of solution of the form of a bound state of two topological solitons known as a breather (also called a bion), that oscillates periodically in time and decays exponentially in space. A breather can play an indeterminate role, i.e., it may cause a parasitic excitations or may be a good generator of electromagnetic waves propagating at frequencies in the terahertz $(\mathrm{THz})$ range. In the homogeneous sine-Gordon system, breathers exist with frequency in the finite plasma band. As our system admits high-frequency band gaps, Josephson junctions with periodic phaseshifts may support breathers with high oscillationfrequency, i.e., gap breathers. It will be interesting to study this type of solution in future investigations.

Another possible future study is also the fact that as pointed out by one of the referees, uniform driving can be unrealistic for long Josephson junctions. Inhomogeneous driving distribution, e.g., as in superconducting films, can radically affect critical currents and lifetimes of the superconductive state [38, 39]. The effect of a nonuniform bias current to the band structure is also addressed as a future study.

\section{Acknowledgement}

We thank the three anonymous reviewers for their careful reading that improved the manuscript.

\section{Appendix A. Constants in the calculations of the Arnold tongues}

The terms $S_{i}$ 's are given by

$$
\begin{gathered}
S_{1}=\frac{1}{2}\left(r_{1}^{2}+\frac{r_{2}^{2}}{4}+\frac{r_{3}^{2}}{9}+\frac{r_{4}^{2}}{16}\right), \\
S_{2}=\frac{1}{8}\left(5 r_{1} r_{2}-\frac{13 r_{2} r_{3}}{9}+\frac{25 r_{3} r_{4}}{16}\right), \\
S_{3}=\frac{r_{1}^{2}}{2}-\frac{5 r_{1} r_{3}}{9}-\frac{5 r_{2} r_{4}}{32},
\end{gathered}
$$

$$
\begin{aligned}
& S_{4}=\frac{r_{1}}{8}\left(5 r_{2}-\frac{17 r_{4}}{4}\right), \\
& S_{5}=\frac{5 r_{1} r_{3}}{9}-\frac{r_{2}^{2}}{8}, \\
& S_{6}=\frac{2 r_{3}}{25}\left[r_{2}-\frac{37 r_{3}}{49}\right]-\frac{r_{1}}{9}\left[r_{1}+2 r_{2}\right] \\
& -\frac{r_{4}}{49}\left[2 r_{3}+r_{4}\right]-\frac{34 r_{2}^{2}}{225} \text {, } \\
& S_{7}=\frac{2 r_{2}}{9}\left[\frac{29 r_{3}}{49}+\frac{8 r_{1}}{25}\right]+\frac{r_{1}}{9}\left[r_{1}+\frac{34 r_{3}}{25}\right] \\
& +\frac{r_{4}}{25}\left[\frac{74 r_{2}}{49}-r 3\right] \text {, } \\
& S_{8}=\frac{r 4}{9}\left[\frac{58 r_{1}}{49}+r_{2}\right]-\frac{r_{1}}{9}\left[r_{1}+\frac{16 r_{2}}{25}\right] \\
& -\frac{248 r_{1} r_{3}}{1225} \\
& S_{9}=\frac{r_{1}}{49}\left[r_{4}-\frac{24 r_{3}}{25}\right]+\frac{r_{2}}{9}\left[r_{2}+\frac{34 r_{1}}{25}\right], \\
& S_{10}=-\frac{2 r_{3}}{25}\left[r_{2}+\frac{37 r_{3}}{49}\right]-\frac{r_{1}}{9}\left[r_{1}-2 r_{2}\right] \\
& +\quad \frac{r_{4}}{49}\left[2 r_{3}-r_{4}\right]-\frac{34 r_{2}^{2}}{225} \\
& S_{11}=\frac{2 r_{2}}{9}\left[\frac{29 r_{3}}{49}+\frac{8 r_{1}}{25}\right]-\frac{r_{1}}{9}\left[r_{1}+\frac{34 r_{3}}{25}\right] \\
& -\frac{r_{4}}{25}\left[\frac{74 r_{2}}{49}+r 3\right] \text {, } \\
& S_{12}=\frac{r 4}{9}\left[\frac{58 r_{1}}{49}-r_{2}\right]+\frac{r_{1}}{9}\left[r_{1}-\frac{16 r_{2}}{25}\right] \\
& +\frac{248 r_{1} r_{3}}{1225} \\
& S_{13}=\frac{r_{1}}{49}\left[r_{4}+\frac{24 r_{3}}{25}\right]-\frac{r_{2}}{9}\left[r_{2}-\frac{34 r_{1}}{25}\right] \text {, }
\end{aligned}
$$




$$
\begin{aligned}
& S_{14}=-\frac{1}{4}\left(\frac{r_{1}^{2}}{4}-\frac{r_{2}^{2}}{9}\right)+\frac{r_{2}}{18}\left(\frac{r_{2}}{2}+r_{4}\right) \\
& +\frac{r_{3}}{8}\left(r_{1}-\frac{5 r_{3}}{8}\right) \\
& S_{15}=\frac{r_{1}}{144}\left(5 r_{2}+13 r_{4}\right)-\frac{r_{3}}{16}\left(\frac{r_{2}}{4}+r_{4}\right) \\
& S_{16}=\frac{r_{1}}{16}\left(r_{1}-\frac{5 r_{3}}{4}\right)-\frac{r_{2}}{36}\left(r_{2}-r_{4}\right), \\
& S_{17}=\frac{r_{1}}{36}\left(\frac{13 r_{2}}{4}-r_{4}\right)-\frac{3 r_{2} r_{3}}{64} \\
& S_{18}=-\frac{1}{4}\left(\frac{r_{1}^{2}}{4}+\frac{r_{2}^{2}}{9}\right)-\frac{r_{2}}{18}\left(\frac{r_{2}}{2}+r_{4}\right) \\
& -\frac{r_{3}}{8}\left(r_{1}+\frac{5 r_{3}}{8}\right) \\
& S_{19}=\frac{13 r_{1}}{144}\left(r_{2}+r_{4}\right)+\frac{r_{3}}{16}\left(3 r_{2}+r_{4}\right), \\
& S_{20}=\frac{r_{1}}{16}\left(r_{1}+\frac{3 r_{3}}{4}\right)+\frac{r_{2}}{36}\left(r_{2}+r_{4}\right), \\
& S_{21}=-\frac{r_{1}}{36}\left(\frac{13 r_{2}}{4}+r_{4}\right)-\frac{3 r_{2} r_{3}}{64} \\
& S_{22}=r_{1}\left(\frac{26 r_{2} r_{2}}{25}-r_{1}\right)+\frac{2 r_{2}}{49}\left(24 r_{3}+\frac{37 r_{4}}{25}\right) \\
& -\frac{r_{3}}{25}\left(24 r_{1}-r_{4}\right) \text {, } \\
& S_{23}=-\frac{2 r_{1}}{25}\left( \pm 13 r_{1}+r_{4}\right) \pm 2 r_{2}\left(r_{1} \mp \frac{25 r_{2}}{49}\right) \\
& -\frac{r_{4}^{2}}{25},
\end{aligned}
$$

$$
\begin{aligned}
S_{24}= & r_{2}\left(r_{2}+r_{3}\right)-\frac{r_{3}}{25}\left(24 r_{1}-r_{4}\right)-\frac{50 r_{1} r_{2}}{49}, \\
S_{25}= & \frac{r_{1}}{25}\left(r_{1}-26 r_{4}\right)+r_{3}\left(r_{1}-\frac{48 r_{2}}{49}\right)+r_{2} r_{4}, \\
S_{26}= & r_{1}\left(\frac{26 r_{2} r_{2}}{25}+r_{1}\right)-\frac{2 r_{2}}{49}\left(\frac{37 r_{4}-24 r_{3}}{25}\right) \\
& +\frac{r_{3}}{25}\left(24 r_{1}+r_{4}\right), \\
S_{27}= & -\frac{2 r_{1}}{25}\left(r_{4}-13 r_{1}\right)-2 r_{2}\left(r_{1}+\frac{25 r_{2}}{49}\right)-\frac{r_{4}^{2}}{25}, \\
S_{28}= & -r_{2}\left(r_{2}-r_{3}\right)+\frac{r_{3}}{25}\left(24 r_{1}+r_{4}\right)-\frac{50 r_{1} r_{2}}{49}, \\
S_{31}= & \frac{1}{4}\left(\frac{r_{2}^{2}}{16}+r_{3}^{2}\right)+\frac{r_{1}}{2}\left(\frac{5 r_{1}}{9} \pm r_{3}\right) \cdot \\
S_{29}= & \frac{r_{1}}{25}\left(r_{1}-26 r_{4}\right)-r_{3}\left(r_{1}+\frac{48 r_{2}}{49}\right)-r_{2} r_{4}, \\
S_{30}= & \frac{2 r_{1}}{9}\left(r_{2}+r_{4}\right)+\frac{r_{3}}{4}\left(\frac{17 r_{2}}{16}+r_{4}\right), \\
= &
\end{aligned}
$$

\section{References}

[1] B. D. Josephson, Possible new effects in superconductive tunnelling, Phys. Lett. 1, 251-253, (1962).

[2] A. Barone, and G. Paterno, Physics and applications of the Josephson effect, (John Wiley \& Sons, New York), (1982).

[3] K.K. Likharev, Dynamics of Josephson junctions and circuits, Gordon and Breach Science Publishers, New York, , (1986).

[4] V.P. Koshelets, S.V. Shitov, L.V. Filippenko, V.L. Vaks, J. Mygind, A.M. Baryshev, W. Luinge, and N. Whyborn, Phase locked 270-440 $\mathrm{GHz}$ local oscillator based on flux flow in long Josephson tunnel junctions, Rev. Sci. Instrum., 71, 289-293, (2000). 
[5] A. Kemp, A. Wallraff, and A.V. Ustinov, Josephson vortex qubit: Design, preparation and read-out, Phys. Stat. Sol. (b) 233, 472-481, (2002).

[6] Y. S. Kivshar, and B. A. Malomed, Dynamics of solitons in nearly integrable systems, Rev. Mod. Phys. 61, 763-915, (1989).

[7] A.V. Ustinov, Solitons in Josephson junctions. Physica D 123, 315-329 (1998);

[8] D.W. McLaughlin and A. C. Scott, Perturbation analysis of fluxon dynamic Phys. Rev. A 18, 1652-1680 (1978).

[9] D. Valenti, C. Guarcello, B. Spagnolo, Switching times in long-overlap Josephson junctions subject to thermal fluctuations and non-Gaussian noise sources, Phys. Rev. B, 89, 214510 (2014).

[10] G. Augello, D. Valenti, A.L. Pankratov, B. Spagnolo, Lifetime of the superconductive state in short and long Josephson junctions, Eur. Phys. J. B 70, 145-151 (2009).

[11] K. G. Fedorov and A. L. Pankratov, Crossover of the thermal escape problem in annular spatially distributed systems, Phys. Rev. Lett. 103, 260601 (2009);

[12] M . Büttiker and R. Landauer, Nucleation theory of overdamped soliton motion, Phys. Rev. A 23, 1397-1410, (1981);

[13] K.G. Federov, A.L. Pankratov and B. Spagnolo, Influence of length on the noise delayed switching of long Josephson junctions, Int. J. Bifurcation and Chaos, 18, 2857-2862 (2008).

[14] C. Guarcello, D. Valenti, A. Carollo and B. Spagnolo, Stabilization effects of dichotomous noise on the lifetime of the superconducting state in a long Josephson junction, Entropy 17, 28622875 (2015).

[15] L.N. Bulaevskii, V.V.Kuzii, and A.A. Sobyanin, Superconducting system with weak coupling to the current in the ground state, JETP Lett., 25, 290-295, (1977).
[16] L.N. Bulaevskii, V.V. Kuzii, A.A. Sobyanin,and P.N. Lebedev, On possibility of the spontaneous magnetic flux in a Josephson junction containing magnetic impurities, Solid State Commun., 25, 1053-1057, (1978).

[17] E. Goldobin, D. Koelle, and R. Kleiner, Semifluxons in long Josephson 0- $\pi$-junctions, Phys. Rev. B, 66, 100508(R), (2002).

[18] J. R. Kirtley, C. C. Tsuei, Martin Rupp, J. Z. Sun, Lock See Yu-Jahnes, A. Gupta, and M. B. Ketchen, Direct imaging of integer and half-integer josephson vortices in high- $T_{c}$ grain boundaries, Phys. Rev. Lett. 76, 1339-1339, (1996).

[19] A. Sugimoto, T. Yamaguchi, and I. Iguchi, Temperature dependence of half flux quantum in $\mathrm{YBa}_{2} \mathrm{Cu}_{3} \mathrm{O}_{7-y}$ tricrystal thin film observed by scanning SQUID microscopy, Physica C, 367, 28-32, (2002).

[20] E. Goldobin, A. Sterck, T. Gaber, D. Koelle, and R. Kleiner, Dynamics of semifluxons in $\mathrm{Nb}$ long Josephson 0- $\pi$ junction, Phys. Rev. Lett., 92, 057005, (2004).

[21] A.V. Ustinov, Appl. Phys. Lett., 80, 3153 (2002).

[22] B.A. Malomed, and A.V. Ustinov, Creation of classical and quantum fluxons by a current dipole in a long Josephson junction, Phys. Rev. B, 69, 069502, (2004).

[23] E. Goldobin, D. Koelle, and R. Kleiner, Ground states of one and two fractional vortices in long Josephson 0- $\kappa$ junctions, Phys. Rev. B, 70, 174519, (2004).

[24] E. Goldobin, H. Susanto, D. Koelle, R. Kleiner, and S.A. Van Gils, Oscillatory eigenmodes and stability of one and two arbitrary fractional vortices in long Josephson 0- $\kappa$ junctions, Phys. Rev. B, 71, 104518, (2005).

[25] H. Susanto, E. Goldobin, D. Koelle, R. Kleiner, and S.A. Van Gils, Controllable plasma energy bands in a one-dimensional crystal of fractional 
Josephson vortices, Phys. Rev. B, 71, 174510, (2005).

[26] K. Vogel, W.P. Schleich, T Kato, D Koelle, R Kleiner, E Goldobin. Theory of fractional vortex escape in a long Josephson junction. Phys. Rev. B, 80, 134515 (2009);

[27] K. Buckenmaier, T. Gaber, M. Siegel, D. Koelle, R. Kleiner, and E. Goldobin Spectroscopy of the Fractional Vortex Eigenfrequency in a Long Josephson $0-\kappa$ Junction. Phys. Rev. Lett. 98, 117006 (2007);

[28] C. Kittel, and P. McEuen, Introduction to Solid State Physics, Wiley, New York, ISBN : 978-0471-41526-8, (1976).

[29] Y. S. Kivshar, and G. P. Agrawal, Optical Solitons: From Fibers to Photonic Crystals, (Academic Press, California, 92101-4495, USA, (2003).

[30] J. Yang, Nonlinear waves in Integrable and Nonintegrable Systems, SIAM, Philadelphia, (2010).

[31] E. I. Rashba, Properties of semiconductors with an extremum loop. 1. Cyclotron and combinational resonance in a magnetic field perpendicular to the plane of the loop, Sov. Phys. Solid State, 2, 1224-1238, (1960).

[32] U.C. Coskun, T.C. Wei, S. Vishveshwara, P.M. Goldbart, and A. Bezryadin, $\{h / e$ Magnetic Flux Modulation of the Energy Gap in Nanotube Quantum Dots, Science, 304, 1132-1134, (2004).

[33] L. N. Trefethen, Spectral Methods in Matlab (Soc. for Industr. \& Appl. Math., Philadelphia, 2000).

[34] A.H. Nayfeh, and D.T. Mook, Perturbation Methods, John Wiley \& sons, New York, (1979).

[35] H. Susanto, Darminto, and S.A. van Gils, Phys. Letts. A 361, 270-276 (2007).

[36] N. W. McLachlan, Theory and Application of Mathieu Functions, Clarendon Press, Oxford, (1947).
[37] A. H. Nayfeh, and D. T. Mook, Nonlinear Oscillations, John Wiley \& sons, New York, (1979).

[38] A. L. Pankratov, Phys. Rev. B 66, 134526 (2002).

[39] K. G. Fedorov and A. L. Pankratov, Phys. Rev. B 76, 024504 (2007). 